$\xi=1$ 娄

\title{
Automated target controlled anaesthesia injector
}

\author{
Sri Vidhya Lakshmi.S ${ }^{1 *}$, Andrews Luxcy. E ${ }^{1}$, Vishnupriya. U ${ }^{1}$, Bhargavi Haripriya. A ${ }^{2}$ \\ ${ }^{1}$ Final year, Biomedical Engineering Department \\ ${ }^{2}$ B.E, M.S Assistant Professor, Biomedical Engineering Department SRM Institute of Science and Technology \\ *Corresponding author E-mail: vidhyaswaminathan.1996@gmail.com
}

\begin{abstract}
Anaesthesia plays a vital role in major operations which lasts for longer duration of time. The anaesthetic condition of a patient is determined by an anaesthetist based on the body conditions and feed the amount of anaesthesia to be delivered into a modern anaesthesia machine, which delivers the drug as instructed. If a patient experience a sudden change in the body condition where the instructed anaesthesia level cannot help, the anaesthetist and doctors will face severe problems during the operation which leads to patient awakening or may even cause death. So, the patient's body condition has to be continuously monitored and the anaesthesia dosage has to be delivered based on the changes in the body condition. This requires an automated operation of anaesthesia injector which works based on the changes in the patient's body condition by monitoring the vital parameters by itself. This can avoid over dosage and its side effects, eventually reduces the death rate caused by the improper administration of Anaesthesia. Initially, certain amount of dosage is delivered by the anaesthetist based on the patient's condition and earlier reports. Then, during the surgery the anaesthesia is administered if required by this 'Automated Target Controlled Anaesthesia Injector'. Our idea is to monitor the necessary body parameters like temperature, blood pressure, and heart rate by the sensors interfacing it to the microcontroller-PIC16f877a and programmed to deliver the dosage level using stepper motor based on the changes in the parameters. Though, there are so many ideas on automated operation of anaesthesia injector using different micro-controllers and microprocessor, the revolution made in this idea is to use PIC Microcontroller which has an advantage of in-built amplifier, analog to digital convertor \& EEPROM coupling with the vital parameters determining the dosage to be administered and assistive mobile-app which helps to reduce the work of anaesthetist by monitoring even from distant places.
\end{abstract}

Keywords: Use about five key words or phrases in alphabetical order, Separated by Semicolon.

\section{Introduction}

Every year, 954 surgeries per daytake place in India [12].General Anaesthesia produces unconsciousness and affects your whole body. Regional anaesthesia affects a portion ofyour body. Local anaesthesia medications are used in many dental procedures, diagnostic tests and some surgeries. They do not cause unconsciousness and are less risky than general 3 anaesthesia. Conscious sedation medications maybe given in addition to local anaesthesia to relax you during a procedure and block memory. Local numbingagents allow you to remain awake and alert while deadening feeling in a small area of your body.Some of the more common side effects that could indicate an anaesthesia overdose are Nausea or vomiting,Respiratorydistress, Hypothermia, Hallucinations, Seizures, Mental or physical impairment, Dementia, Prolonged unconsciousness, Brain injuries and even Death.

During the surgery, anaesthetic condition of a patient is highly essential. An anaesthetist plays a key role in observing the earlier reports and body conditions of the patient for assisting the anaesthesia dose. Anaesthesia cannot be given completely to a patient in a single stroke, which may cause severe side effects and even leads to death. There are also high chances of patient awakening in-between the surgery, if the dosage delivered is low.

Hence, the dosage of anaesthesia should be administered to a patient based on observing earlier reports by the anaesthetist before undergoing the surgery and also deliver whenever there are changes found in the body parameters during the surgery which will be a difficult task for an anaesthetist.
To overcome the difficulties faced by the Anaesthetist, An automated operation of anaesthesia injector is highly encouraged by anaesthetists.

The anaesthesia dose to be delivered during the surgery is based on the changes in body conditions of the patient which can be observed by monitoring the vital parameters. The important parameters by which the dosage can be determined are Body temperature, Heart-Rate and Blood pressure. So, The Anaesthesia Injector has to work based on the changes in the vital parameters of the patient to deliver accurate amount of dosage. This can absolutely avoid death rate caused by over dosage of anaesthesia and also its side effects.

This project is into automated operation of Anaesthesia Injector by monitoring the vital parameters of the patient which requires a Heart-beat sensor, Temperature sensor, Blood pressure sensor and an interface PIC microcontroller to couple the sensors and the injector operation. This idea is also into developing an assistive Mobile-app which provides user friendly interface to the anaesthetist. Suppose if an anaesthetist is an emergency to assist two or more surgery at the same time, he can monitor all the three surgery at his mobile and attend the one he is require to.

The revolution made in this idea is to use PIC Microcontroller which has an advantage of in-built amplifier, analogue to digital convertor \& EEPROM coupling with the vital parameters determining the dosage to be administered and assistive mobileapp.This paper further explains the Working of the system with Block diagram description, Measurement of biomedical parameters, PIC as an interface, System architecture which includes hardware implementation and component selection. 


\section{Objectives}

Objectives of this prototype include:

- A working model of automated anaesthesia injector used for a patient during the surgery.

- To deliver accurate amount of dosage to the patient automatically by monitoring the vital parameters which will avoid over dosage and its side effects.

- To gain wide knowledge in embedded systems, which makes it to work very compactly.

- To develop an assisting mobile app which provides user friendly interface to the anaesthetist.

\section{Working of the system}

\subsection{Block diagram description}

The below mentioned block diagram represents the working of the automated target controlled anaesthesia injector. By using the default value provided along with the Microcontroller, the anaesthetist can set the level of anaesthesia to be administered to the patient in terms of milli-litres per hour. It analyses various vital parameters from the various sensors. Then it determines the direction of rotation of DC motor. The rotation of DC motor causes the movement of infusion pump either in forward or backward direction and the anaesthesia provided in the syringe is injected into the patient body. Micro controller is programmed with the certain ranges based on patient body condition through which the motor plays the role. If suppose the patient conditions goes above those certain ranges then the motor will run and inject the Propofol which is the general anaesthesia commonly used for all the patients. If the values of vital parameters goes below the given range then motor stops running and gives an alarm so as to indicate the anaesthetist, in case of emergency. Micro controller is programmed using two software namely, MPLAB IDE v8.92 and Proteus 8 Professional. Biomedical parameter ranges can be programmed using MPLAB and those programs can be executed using Proteus software. The hardware circuit can also be interfaced with the PC using the TTL cable.

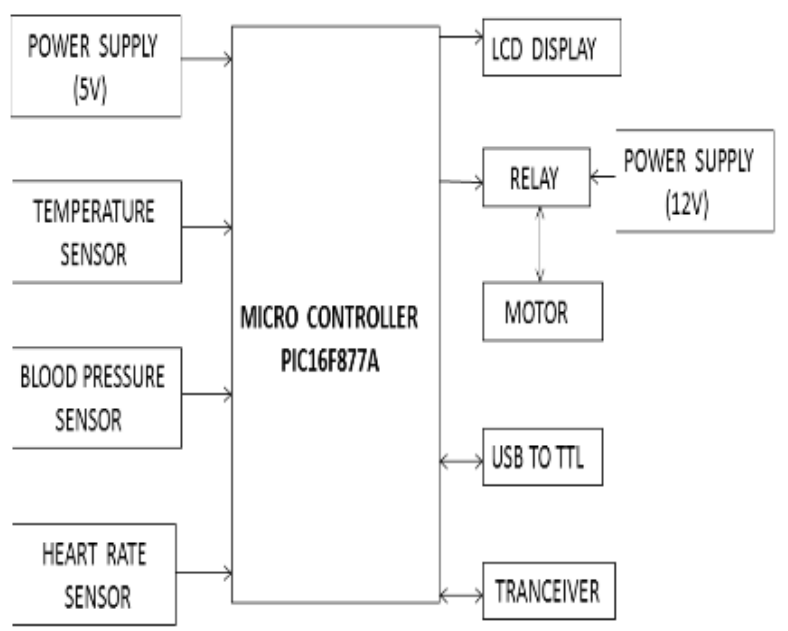

Fig. 1: Block Diagram Representation of the Automated Anaesthesia Injector.

\subsection{Measurement of biomedical parameters}

In the proposed design, biomedical parameters such as blood pressure, heart rate and temperature are taken into consideration, they depict the patient's overall condition. These are the three vital parameters that are monitored while administering anaesthesia Biomedical sensors are used in order to measure them, based on the output from these sensors the amount of anaesthesia to be injected is determined. The whole system functions depend on these parameters, especially the motor. Mostly biomedical sensors are transducers and thermistors which are fabricated in specific forms suitable to use. The sensors used for measuring the vital parameters are,Blood pressure - The normal blood pressure range is $120 / 80 \mathrm{mmHg}$, after the injection of anaesthesia the individuals blood pressure may increase or decrease but once the patient becomes unconscious, the blood pressure comes down from the normal range and this condition is termed as hypotension Heart beat - The heartbeat of an unconscious patient is also below the normal rate of 60 to $100 \mathrm{bpm}$. Photoplethysmography is a technique where the light absorption of the blood vessels in finger tip is taken in consideration to reveal the heartbeat rate, as there will be fluctuation in the reflected light due to the pulsatile flow caused by the heartbeat.

Temperature sensor- The temperature of a patient after anaesthesia administration enters mild hypothermia state but this is very slightly below the normal state of 36 degrees, if the body temperature decreases less than 35 degrees then the anaesthetist is alarmed.

\subsection{PIC as an interface}

The revolution made in this idea is to use PIC Microcontroller as an interface which has an advantage of in-built amplifier, analog to digital convertor \& EEPROM coupling with the vital parameters which helps to determine the dosage to be administered.The blood pressure sensor, temperature sensor, and the heart rate sensor act as inputs to the microcontroller. Basically analogue signals are too weak and of low frequency which is difficult for further processes, so an in-built amplifier is used for amplification. The amplified analogue signal is converted into digital signal by inbuilt analogue to digital convertor of PIC. Normal range of values for each vital parameter is programmed in the microcontroller and also programmed in such way to run the motor in order to administer the drug when the parameter goes out of the normal range. This PIC microcontroller is chosen as interface as it has got multipurpose to do with as an in-built amplifier, analogue to digital convertor \& EEPROM.

\subsection{System architecture}

In our proposed system microcontroller plays a key role in executing the output. The sensors which are connected with the microcontroller senses the value of biomedical parameters and read the inputs from the keypad provided with the microcontroller into the software, MPLAB. Proteus software executes the output based on the program which we implemented in MPLAB. The outputs of the circuits is amplified by means of an amplifier and fed into an analog to digital converter. The microcontroller displays the parameters in digital value in the display device. The software is mainly used to burn the programs in MPLAB and will operate in the Proteus. The parameters are checked with their values for every specified interval. These software calculate the stepper motor movement with the parameters provided by the sensors.

\subsection{Software implementation}

The block diagram is produced in the software by connecting the circuit using the selected components which are available readily in the Proteus 8 Pro. The normal ranges of all the body parameters are fed in the microcontroller along with the conditions to make the motor run when the sensor output goes out of the normal range with the help of algorithm made in the MP Lab software. 


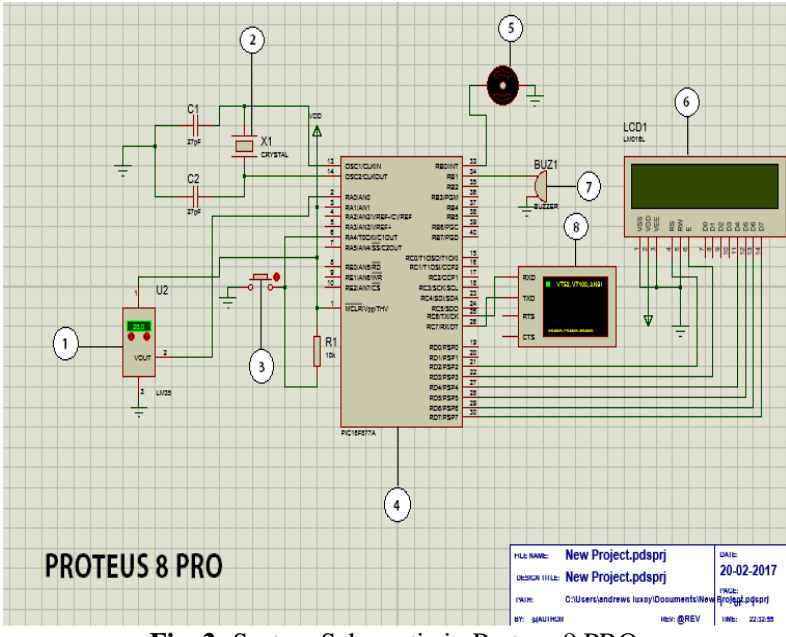

Fig. 2: System Schematic in Proteus 8 PRO.

In FIGURE. 2 the labelled parts are,

1) Temperature sensor

2) Crystal oscillator

3) Heart rate sensor

4) PIC16F877A

5) DC Motor

6) LCD Display

7) Buzzer

8) Blood pressure sensor

The working of the setup is explained in experimental methodology.

Algorithm designed for the functioning of PIC Microcontroller with the help of MPLab:

Step 1: Connect the sensors to the patient

Step 2: Start the automated anaesthesia injector

Step 3: If 'TEMP' is $>36$ [high]

Output value $=1$

(Motor will start rotate to inject the gas)

Step 4: Else if 'TEMP' $=<35$ [low]

Output $=0$

(Motor off \& buzzer on)

Step 5: If 'BP' $=>120 / 80 \mathrm{~mm}$ hg [high]

Output $=1$.

(Motor will start rotating)

Step 6: Else if 'BP' $=<120 / 80 \mathrm{~mm}$ hg [low]

Output $=0$

(Motor off $\&$ buzzer on)

Step 7:If 'HR' $=>100$ beats $/ \mathrm{min}$ [high]

Output $=1$.

(Motor will start rotating)

Step 8:Else if 'HR' $=<60$ beats/min [low]

Output $=0$.

(Motor off \& buzzer on)

\subsection{Hardware implementation}

The system architecture designed in the software is generated as such in hardware with the same specifications as produced in software. The hardware components are chosen in such a way to be compatible with the PIC microcontroller. The components selection and specifications of the system architecture are listed below.

Components used in automated target controlled Anaesthesia Injector are,

- Regulated power supply (5V and $12 \mathrm{~V})$

- Microcontroller (PIC16F877A)

- Blood pressure sensor

- Heart rate sensor (TCRT1000)

- Temperature sensor (LM35)

- $10 \mathrm{rpm} 12 \mathrm{~V}$ DC motor

- LCD display

\section{- USB \& TTL}

Regulated power supply

Power supply is the primary component, it consists of a transformer, bridge rectifier and a filter. Voltage regulator IC's such as LM7805 and LM7812 are used in combination with capacitors. The bridge rectifier converts the $\mathrm{AC}$ voltage to $\mathrm{DC}$ voltage. The main AC voltage of $230 \mathrm{~V}$ is given to the two step-down transformers, one step-downs $230 \mathrm{~V}$ to $5 \mathrm{~V}$ which is used for the PIC microcontroller and the other for converting $230 \mathrm{~V}$ to $12 \mathrm{~V}$ for the DC motor, a relay circuit is used in this case to control the power supply.The regulated power supply $5 \mathrm{v}$ \&amp; $12 \mathrm{v}$ circuit board was manufactured in India and supplied by Surya electricals.[13]

Microcontroller (PIC16F877A)

The main component of this idea is PIC-16F877A

It's a 40-pin 8-bit CMOS FLASH microcontroller.

It has two types of internal memories:program memory and data memory.Program memory is provided by $8 \mathrm{~K}$ words of flash memory.Data memory has two sources: One type of data memory is 368 byte RAM, and other is 256byte

This microcontroller acts as an interface between biomedical parameter sensors and the output to be delivered.Pic microcontroller 16 f877a used is supplied by India mart which is manufactured in India.[14]

Blood pressure sensor

The normal blood pressure range is $120 / 80 \mathrm{mmHg}$, after the injection of anaesthesia the individuals blood pressure may increase or decrease but once the patient becomes unconscious, the blood pressure comes down from the normal range and this condition is termed as hypotension. The transducer used for measuring blood pressure is the common ones that are found in the multiparameter monitors which contains the standard cuff and pump, this setup is connected to a pressure sensor that measures the patient's blood pressure and sends it to the pic microcontroller. When there is any change or increase in the patient's blood pressure then the system is designed to alert the anaesthetist and the motor runs to administer anaesthesia in case of high blood pressure which indicates the patient is regaining his/her consciousness.

Heart beat sensor

The heart beat sensor used here is TCRT1000, it is a reflective optical sensor which works on the principle of photoplethysmography. The heartbeat of an unconscious patient is also below the normal rate of 60 to $100 \mathrm{bpm}$. The motor runs when the heart beat is above normal range as it indicates the patient is awake. Photo plethysmography is a technique where the light absorption of the blood vessels in finger tip is taken in consideration to reveal the heartbeat rate, as there will be fluctuation in the reflected light due to the pulsatile flow caused by the heartbeat. In TCRT1000 there are two optical components an IR or LED emitter and detector, both are placed on the same side of the finger as it follows reflective technology, the whole system is packed in a light blocking package to avoid wrong output.Heart rate sensor TCRT1000 was made in India supplied by SRI electronics, embedded solutions. [15].

Temperature sensor

The temperature of a patient after anaesthesia administration enters mild hypothermia state but this is very slightly below the normal state of 36 degrees, if the body temperature decreases less than 35 degrees then the anaesthetist is alarmed. The temperature sensor used is LM32 is a linear integrated circuit which gives its output directly in Celsius scale which reduces further calculations as if it is Kelvin then subtraction of large numbers are involved. Temperature sensor LM35 was made in India supplied by amazon with the model robokart. [16]

DC motor-10RPM $12 \mathrm{~V}$ with Gearbox

The features of this DC motor are 3000RPM base motor, $6 \mathrm{~mm}$ shaft diameter with internal hole, $125 \mathrm{gm}$ weight,Same size motor available in various $\mathrm{rpm}, 12 \mathrm{kgcm}$ torque,No-load current $=60$ $\mathrm{mA}(\operatorname{Max})$, Load current $=300 \mathrm{~mA}(\operatorname{Max})$. The motor runs, instructed by the PIC microcontroller when it finds changes in the normal range of parameter values.DC motor was manufactured in 
India and supplied by Robotic kits and accessories through amazon.[17]

LCD Display

LCD display which we are using in our proposed system is LCD $16 * 2$ which contains 16 characters by 2 line display that runs at $3.3 \mathrm{~V}$ utilizes the common ST7066/HD44780 parallel interface(datasheet).Itincludes white LED backlight, $5 * 8$ dot characters and $1 / 16$ duty cycle. We need 11 general $\mathrm{I} / \mathrm{O}$ pins tointerface to this LCD screen. The purpose of the controller is to generate pixel patterns and driveindividual pixels of the LCD to show characters and symbol. Common modules works with 5v supply. Pin

1 is connected to GND and pin 2 is connected to $+5 \mathrm{v}$ supply. There are three control lines namely the

$\mathrm{RS}, \mathrm{R} / \mathrm{W}, \mathrm{E}$. these are connected to microcontrollers general purpose input output line.DB0 TO DB7 alsoconnected to microcontroller general purpose input output line.LCD display was manufactured in India and supplied by eBay.[18]

USB to TTL

USB to TTL serial cable which is used to connect TTL interface devices to USB. The I/O pins of this cableconfigured to operate at $3.3 \mathrm{~V}$. This cable is perfect for powering, and connecting .The USB side ofthe cable is USB powered and USB 2.0 full speed compatible. Each cable is $1.8 \mathrm{~m}$ long and supports adata transfer rate up to $3 \mathrm{Mbaud}$. TTL-232R cable requires USB drivers, which are used to make theFT232R in the cable appear as a virtual COM port. This then allows the user to communicate with theUSB interface via a standard PC serial emulation port.USB cable was manufactured in India and supplied by eBay.

\section{Experimental methodology}

We successfully implemented the hardware circuit as per the block diagram after the confirmation of working in the Proteus software. Then proceeded with experimenting the hardware with few subjects by connecting the heart rate sensor to the tip of the finger, blood pressure cuff is tied to the left hand of the subject and we made the patient to hold the temperature sensor. The outputs of the sensor such as the temperature, pulse rate, blood pressure and seconds can be seen in the LCD display and the output is given to the PIC microcontroller also where the sensor outputs are matched with the algorithm given to the controller and noted the change in infusion by the running of motor. The motor runs only when there is changes in the normal values of the body parameters. When the value changes the motor starts running automatically to inject the anaesthesia dosage. The performance of the automated anesthesia Injector was checked for different biomedical parameters. The device was checked whether it runs according to the code written on the microcontroller.

Table 1: Device Performance

\begin{tabular}{|l|l|l|}
\hline $\begin{array}{l}\text { BIOMEDICAL } \\
\text { PARAMETERS }\end{array}$ & $\begin{array}{l}\text { LEVEL OF } \\
\text { PARAMETERS }\end{array}$ & $\begin{array}{l}\text { MOTOR } \\
\text { STATE }\end{array}$ \\
\hline Blood pressure & High & Motor Runs \\
& Normal, Low & No Change \\
\hline Heart Beat & High & Motor Runs \\
& Normal, Low & No Change \\
\hline Temperature & High & Motor Runs \\
& Normal, Low & No Change \\
\hline
\end{tabular}

\section{Analysis \& result}

On the whole, it is apparently seen that monitoring of the vital parameters using the heart sensor, temperature sensor and blood pressure sensor gives more accurate measure of delivering the anaesthesia. This model resulted in incorporating the values of the sensors output with the values stored in PIC microcontroller to function the motor and deliver appropriate amount of anaesthesia, The Transceiver attached with the model helps to monitor the dosage level induced by developing a mobile app. This automated operation of anaesthesia injector reduces the side effects and deaths caused due to the improper dosage of anaesthesia.

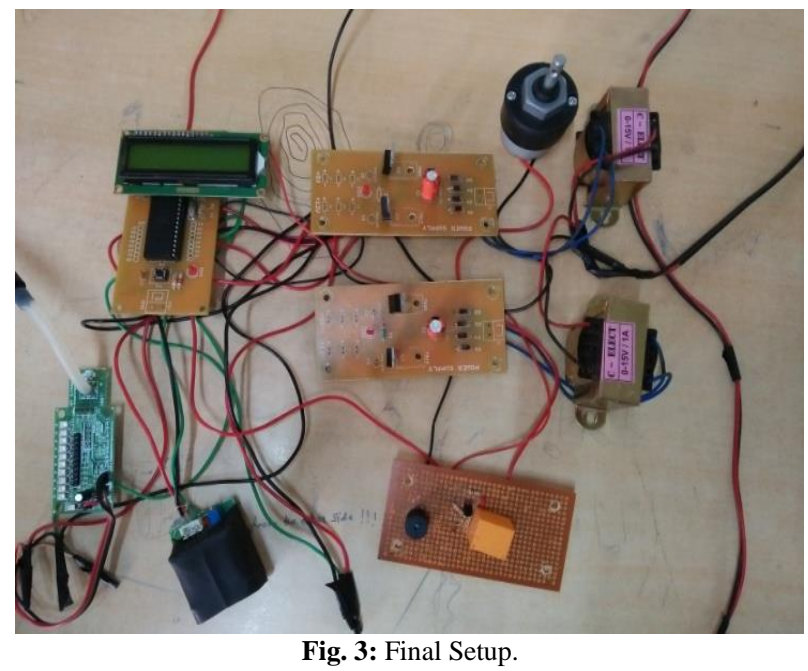

\section{Future Enhancements}

- The size and cost of the equipment can be reduced.

- Many more biomedical parameters can be included such as respiration, retinal size, age, weight and height for more accurate deliver of dosage.

\section{Conclusion}

Modern technology is applied to automate the anaesthesia induction process, it makes the work of the anaesthetist easy and lets him to concentrate on other critical issues. Automated anaesthesia injector is also accurate and safe in case of longer surgical hours. The system is on the whole automatic and effective, as vital biomedical parameters such as heart beat, blood pressure and temperature are simultaneously measured to give an end result continuously. Future improvements like adding intelligence to the system to specify exact amount of anaesthesia to be delivered to patients of various age groups can be created.

\section{Acknowledgment}

We would like to thank the Department of Biomedical Engineering, SRM Institute of Science \& Technology for helping us throughout the project by letting us choose our own title and finish this project successfully.

\section{References}

[1] Computerized Anaesthesia infusion system (International journal of Electrical, electronics and computer systems-IJEECS) 2014

[2] Automated Anaesthesia delivery pump (IOSR journal of pharmacy and biological sciences) 2014.

[3] Low cost anaesthesia injector based on arm processor (International journal of advanced research in computer and communication engineering 2013).

[4] Anaesthesia Regularization using heart beat sensor (AR Digital International Journal of engineering, education and technology ARDIJEET 2014)

[5] Microcontroller based anaesthesia injector, Rana Muhammad Shakeel.

[6] Bio medical Instrumentation and Application, William John Webster. 
[7] Microcontroller and their applications- Kenneth J.Ayalaa - Pen ram International.

[8] David A Bell "Electronic Devices \& Circuits" [9] Temperature sensor,
http://www.electronicslovers.com/2014/11/temperature-monitorusing-pic16f877a.htmlaccessed on $11^{\text {th }}$ January, 2018

[10] LCD Display, https://electrosome.com/lcd-pic-mplab-xc8/accessed on $11^{\text {th }}$ January, 2018

[11] Complications of anaesthesia, https://www.bakerandgilchrist.com/medical-malpracticelawyer/complications-of-general-anesthesia/accessed on $11^{\text {th }}$ January, 2018

[12] Surgeries

in India, https://data. worldbank.org/indicator/SH.SGR.PROC.P5?end=2012 \&locations $=I N \&$ start $=2012 \&$ view $=$ chart $\&$ year $=2015$ accessed on $11^{\text {th }}$ January, 2018

[13] Regulated power supply, https://www.flipkart.com/surya-el-5v$12 \mathrm{v}$-regulated-power-supply-board/p/itmepmwgbz6ex3znaccessed on $11^{\text {th }}$ January, 2018.

[14] PIC

Microcontroller https://dir.indiamart.com/search.mp?ss=pic+microcontroller+board +16 f877a\&amp; src=ascontext $\% 3 \mathrm{Akwd} \% 3 \mathrm{Dpic}+$ microcontrolleraccessed on $11^{\text {th }}$ January, 2018

[15] Heart beat sensor, https://dir.indiamart.com/impcat/heart-beatsensors.htmlaccessed on $11^{\text {th }}$ January, 2018

[16] Temperature sensor, https://www.amazon.in/LM-35-TemperatureSensor-Robokart/dp/B00ZNCBQ9O?tag=googinhydr18418-

21\&amp;tag=googinkenshoo-21\&amp;ascsubtag=d3fb631b-b2d1412d-b343-730e4074b6bcaccessed on $11^{\text {th }}$ January, 2018

[17] Motor, https://www.amazon.in/10-RPM-Geared-MotorRobokart/dp/B010V2JX68accessed on $11^{\text {th }}$ January, 2018

[18] USB to TTL cable, https://www.ebay.in/itm/6Pin-FTDI-FT232RLUSB-To-Serial-Adapter-Module-USB-TO-TTL-RS232-ArduinoCable-/222771214743?_trksid=p2059707.m48543.19013, accessed on $11^{\text {th }}$ January, 2018. 